\title{
Anglicismos léxicos en la prensa italiana, francesa y española
}

\author{
Gisella Policastro Ponce \\ Universidad de Córdoba \\ gisella.policastro@uco.es \\ https://dx.doi.org.10.12795/futhark.2019.il4.09
}

Fecha de recepción: 10.03 .2018

Fecha de aceptación: 13.06.2019

Resumen: El presente trabajo aborda el estudio de los anglicismos léxicos presentes en los textos de índole periodística en italiano, francés y español. Para ello, se ha elaborado un corpus con los textos periodísticos más destacados durante el año 2018 en la prensa italiana, francesa y española, se han extraído los préstamos lingüísticos procedentes del inglés $y$, en las páginas siguientes, se llevará a cabo un análisis contrastivo al objeto de considerar la presencia de dichos préstamos léxicos en cada lengua.

Palabras clave: anglicismo, préstamos lingüísticos, textos periodísticos, lexicología.

\section{Lexical Anglicisms in the Italian, French and Spanish Press}

Abstract: This paper deals with the study of lexical anglicisms which are in journalistic texts in Italian, French and Spanish. For this purpose, a corpus has been developed with the most outstanding journalistic texts during the year 2018 in the most relevant newspapers in Italy, France and Spain. Then, the semantic loans from English have been extracted and, in the following pages, a contrastive analysis will be carried out in order to consider the presence of these lexical loans in each language.

Keywords: Anglicism, language loans, journalistic texts, lexicology.

Sumario: I. Introducción. 2. Metodología. 3. Análisis de resultados. 3.I. Italia. 3.2. Francia. 3.3. España. 3.4. Análisis contrastivo. 4. Conclusión. 


\section{Introducción}

De acuerdo con la corriente del relativismo lingüístico, el lenguaje es el reflejo de cómo interpretamos la realidad que nos rodea; una idea que queda recogida en la hipótesis débil' de Sapir-Whorf (1975) que se fundamenta en el siguiente principio: "existe relación entre el lenguaje que una persona utiliza, y la forma en que percibe el mundos. A pesar de que este planteamiento ha generado muchas críticas, si se analiza desde su interpretación débil, no cabe duda de que el lenguaje tiene en sí mismo nos aporta información sobre una sociedad, una realidad, una cultura. ¿O, por el contrario, como presenta la Sociolingüística, son los factores extralingüísticos los que influyen y condicionan los diferentes registros lingüisticos? Dicha reflexión nos lleva a prestar atención a la sociedad contemporánea occidental, y a identificar en qué términos hace uso del lenguaje.

Hoy en día, la incursión de lo anglosajón como lingua franca (Crystal, 2003) y como cultura franca, tanto a nivel europeo como internacional, se ha convertido en un hecho fehaciente $y$ en un requisito indispensable para vivir en nuestra sociedad actual. Como lingua franca, porque representa hoy en día el idioma de comunicación referente en el mundo; y como cultura franca ${ }^{2}$, porque poco a poco se ha ido adentrando en nuestros hogares, y hemos adaptado a nuestra cultura diferentes costumbres propias de países de habla inglesa. Como sugiere SnellHornby (2000: 16) «US culture is to the cultura franca of the present time what the English language is to English as a lingua franca», es decir, que asociamos la lengua franca a la lengua inglesa, hoy en día, y particularmente la cultura franca a la cultura americana $o$ estadounidense.

El fenómeno dinámico de la globalización, que comenzó a desarrollarse en el Mundo Occidental durante la segunda mitad del siglo XX, "eligió" el inglés como idioma mundial, potenciando de esta forma su expansión como lengua de comunicación a nivel internacional. Desde un punto de vista sociolingüístico, este fenómeno global genera un impacto en la estructura social, la dimensión cultural y el desarrollo lingüístico, fortaleciendo y favoreciendo a aquellos estados supranacionales que tienen un mayor peso dentro del ámbito político, económico, y/o científico. La primacía de países como Estados Unidos en sectores tan relevantes para la sociedad como la innovación científica y técnica se traduce en la

\footnotetext{
${ }^{1}$ La hipótesis de Sapir-Whorf sobre el relativismo lingüístico presenta dos versiones: una versión fuerte y una versión débil.

2 Snell-Hornby (1999) fue la primera en utilizar el término, e inspirada en sus estudios, Zethesen (2010: 549) propone dos definiciones de este término: «I. a common culture existing in a discourse community and thereby facilitating communication between members of this community and by extension from lingua franca: 2 . a globalised common culture which transgresses discourse communities and which eliminates the existence of cultural barriers in international communication (including translation)».
} 
producción literaria de artículos, revistas, libros $y$, en términos generales, divulgación del conocimiento y de nuevos resultados en su lengua materna, lo que implica la aparición indiscutible de neologismos en dicho idioma.

En lo que respecta a otros ámbitos, como el comercio, las relaciones internacionales, la moda, el deporte o la economía, el inglés se ha erigido como herramienta de comunicación, siendo habitual que tanto transacciones, interrelaciones comunicativas como documentos se redacten en dicha lengua. El monopolio del inglés se ha visto favorecido igualmente por las Nuevas Tecnologías de la Información y de la Comunicación en lo que concierne el modo de interactuar entre las personas ha sufrido una transformación, tanto en medio como en forma, existiendo un flujo de información que crece exponencialmente y que crea una necesidad en el usuario, ya no solo por estar en constante comunicación con los demás, sino por utilizar indiscutiblemente esa lengua y no otra, esos términos y no otros. Asimismo, los medios de comunicación representan hoy en día el principal recurso de expansión de la información en una «aldea global» (McLuhan, 1962) caracterizada por la instantaneidad, y la inmediatez en todos los aspectos de la vida social, económica, política, cultural y lingüística. Nuestro estilo de vida, la toma de decisiones a la hora de adquirir o consumir determinados productos, nuestras costumbres, se ven influenciados por los medios de comunicación (masiva) que afectan a y actúan sobre nuestra forma de pensar, de actuar y de expresarnos. Entre los medios de comunicación, la prensa se presenta como un fenómeno social influyente que, tal y como se ha puesto de manifiesto a través de diversos estudios, determina el comportamiento y el lenguaje de la sociedad, y de manera simultánea refleja la forma en que la sociedad vive y se comunica.

Los medios de comunicación $y$, en concreto, la prensa, juegan, especialmente hoy en día, un papel de primer orden en la difusión de los préstamos lingüísticos, en particular del inglés porque contribuyen de manera fundamental a la reducción de las distancias y la conexión directa y constante entre diferentes partes del mundo. Todo ello, según Matras (2013: 10), con el siguiente propósito: «to make optimal use of the full range of expressive structures within the linguistic repertoire that is at their disposal and regards individual speakers' creativity in discourse as a major trigger for long-term language change».

El objetivo de esta investigación comprobar la presencia de anglicismos localizados en diversos periódicos más populares de la prensa italiana, francesa y española. 


\section{Metodología}

El presente trabajo tiene por objeto el análisis comparativo de la importación léxica del inglés en lenguas romances como el italiano, el francés y el español en los medios de comunicación contemporáneos, concretamente en la prensa digital. La hipótesis que se plantea es la siguiente: a) la evidencia de que existe actualmente una presencia importante de préstamos léxicos en estos tres idiomas, centrando la atención exclusiva en los anglicismos; b) es posible identificar los contextos en los que se utiliza con mayor intensidad este tipo de palabras y asociarlo a las áreas de conocimiento en las que los países de habla inglesa tienen mayor peso.

La metodología llevada a cabo se estructura de la siguiente forma:

I. Se elaboró un corpus textual basado en los cuatro periódicos más importantes de cada país objeto de estudio y pertenecientes a diferentes áreas temáticas.

\begin{tabular}{|l|l|l|l|}
\hline \multicolumn{1}{|c|}{ Países } & ITALIA & FRANCIA & ESPAÑA \\
\hline $\begin{array}{l}\text { Información } \\
\text { general }\end{array}$ & Corriere della Sera & Le Parisien & El Mundo \\
\hline $\begin{array}{l}\text { Información } \\
\text { general }\end{array}$ & La Repubblica & Le Monde & El País \\
\hline $\begin{array}{l}\text { Información } \\
\text { económica y de } \\
\text { negocios }\end{array}$ & II sole 24 ore & Les Échos & El Economista \\
\hline $\begin{array}{l}\text { Información } \\
\text { deportiva }\end{array}$ & $\begin{array}{l}\text { La Gazetta dello } \\
\text { Sport }\end{array}$ & L'Équipe & Marca \\
\hline
\end{tabular}

Tabla I. Clasificación por temática de los periódicos consultados. (Elaboración propia)

2. Dentro de cada uno de estos periódicos, se recopilaron las noticias más relevantes de este último año 2018.

3. Se realizó una búsqueda manual de los anglicismos presentes en cada texto periodístico publicado en dichos periódicos y durante dicho periodo.

A raíz de los resultados obtenidos para cada lengua, y tras una contextualización breve de la investigación llevada a cabo, se presentará un análisis contrastivo en el que se identificarán las particularidades más representativas derivadas de la presencia de anglicismos en el marco periodístico de cada lengua. 
En relación al punto I, nos gustaría aclarar que la elección de dichos periódicos se ha basado en la consulta a través de la red sobre los diarios más leídos en cada país. Otro parámetro para tener en cuenta en esta selección fue la temática, puesto que se optó por incluir en la muestra de cada país dos diarios de temática general y diferente perfil ideológico, considerándose la posible existencia de diferencias entre los lineamientos, principios y valores de cada periódico, que son los que rigen la actividad periodística y definen el modo en que se abordan los hechos, el lenguaje y/o la temática. Los otros dos periódicos restantes pertenecen, en cada caso, al ámbito económico y deportivo, en la medida en que hemos estimado que pueden ser las áreas de especialidad que recurran con mayor frecuencia al uso de anglicismos.

En lo que respecta al periodo seleccionado, se pretende tomar como referencia los resultados más actuales, por este motivo se ha tomado el muestreo exclusivamente del año 2018 y de aquellas noticias que fueron más leídas o tuvieron un mayor impacto por suscitar mayor interés entre los lectores, en tanto que no debemos olvidar que «la prensa es reflejo de la realidad vivida en un determinado periodo de tiempo» (Vázquez Amador, 2015).

Por último, la extracción terminológica de los préstamos léxicos procedentes del inglés se efectuó de forma manual por razones evidentes, puesto que no es posible realizarlo de forma automática.

\section{Análisis de los resultados}

Atendiendo a la bibliografía existente en torno a esta temática y siguiendo los métodos de análisis de resultados desarrollados por otros autores en estudios similares, procedemos a exponer los resultados obtenidos.

En el primer nivel de análisis individualizado, comenzaremos con una breve reseña sobre la presencia de préstamos léxicos procedentes del inglés en cada lengua; seguidamente presentaremos la extracción terminológica de anglicismos llevada a cabo por idiomas en cada periódico, acompañada de un breve comentario acerca de las conclusiones que se evidencia de dichos datos.

\section{I. Italia}

Con motivo de las relaciones comerciales con mercaderes italianos, el inglés ha estado en contacto con esta lengua romance desde el siglo XII (Cartago, 1994), existiendo por entonces precisamente una mayor influencia del italiano sobre el inglés. Una interferencia que cambió de rumbo a partir de la Revolución Industrial, el crecimiento del poder económico y colonial del imperio británico, que fomenta la introducción e implantación léxica de vocablos ingleses a través del francés, que 
se mantuvo hasta el siglo $X X$ como la lengua extrajera de referencia en Italia. Posteriormente, sobre todo a partir de la segunda mitad del siglo $X X$, tras la Segunda Guerra Mundial, la cultura angloamericana comenzó a expandirse en el mundo europeo y adentrarse en prácticamente casi todos los ámbitos de la vida cotidiana occidental, ocupando un lugar hegemónico en la cultura y, por ende, en la lengua. En el caso de Italia, en particular, se concedió un reconocimiento de superioridad a la cultura estadounidense (como país aliado) que quedó de manifiesto a través de la lengua. El signo de imperialismo lingüístico (Phillipson, 1998, 2008; Hagège, 2002) que manifiesta hoy en día el inglés, ha adquirido un cierto grado de prestigio por parte de los hablantes italianos, que consideran el uso de anglicismos como «préstamos de lujo» (Italiano, 1999), que, como define Coco (2003: 44), se utilizan «per ottenere effetti stilistici ed espressivi, per darsi un tono, per snobismo, per il prestigio accordato ad un data civiltà e cultura.»

Una muestra de ello se puede apreciar en la siguiente tabla que recoge los resultados de nuestra investigación:

\begin{tabular}{|c|c|c|c|}
\hline II Corriere della & La Repubblica & II Sole 24 ore & La Gazetta dello sport \\
sera & & & \\
\hline audit & audience & benefit & alert \\
authority & best practices & big data & assist \\
award & brand & business & basket \\
badge & break & bonus & best \\
background & burning & budget & bomber \\
cameraman & drive player & cash & club \\
card & exit poll & center & coach \\
challenge & exit strategy & check copy & competitor \\
fan & load & coach & cup \\
fashion & location & data point & derby \\
feeling & mail & digital edition & fair-play \\
form & mood & extra-time & highlights \\
leader & news & import & hobby \\
meeting & location & input & Knock out \\
mission & mood & jobs & killer \\
must & news & killer & leader \\
network & on air & load & manager \\
news & performance & mail & match \\
on line & premier & mini-holding & mister \\
open & privacy & mission & network \\
outing & scoop & off-shore & plaoff \\
partner & self-control & paywall & punch \\
prospect & standing ovation & record & record \\
report & snob & scouting & running \\
wireless & weekend & & Futhark I4 (20I9) \\
\hline I44 & &
\end{tabular}




\begin{tabular}{|l|c|c|c|}
\hline leaflet & & size & set \\
& & states & show \\
& & standard & shock \\
& & surplus & test \\
& & rumors & track \\
& & working & \\
& welfare & \\
\hline
\end{tabular}

Tabla 2. Anglicismos presentes en las noticias más relevantes del Corriere della sera, la Repubblica, il Sole 24 ore y la Gazetta dello Sport durante el año 2018 (Elaboración propia)

\subsection{Francia}

El fenómeno del anglicismo presenta sus primeras menciones en Francia durante el siglo XVIII, considerada una «façon de parler Angloise» (Richelet 1732: 88). La anglomanía que fue duramente criticada por la sociedad francesa, mientras que, en Canadá, al estar expuesto a un entorno multilingüe, se convirtió en un fenómeno de pleno derecho. Al igual que en Italia, el país galo experimentó también una mayor invasión del «emprunt à l'anglais» en el lenguaje cotidiano y especializado a partir de la segunda mitad del siglo $X X$. Sin embargo, no fue tan aceptado en la cultura francesa como en la italiana, de hecho, no tardaron en buscarse medidas institucionales para controlar el anglicismo. Entre ellas, la ley BasLauriol (Loi $n^{\circ} 75$ - I349 du 3 I décembre 1975 relative à l'emploi de la langue française) - la ley Toubon (Loi $n^{\circ}$ 94-665 du 4 août 1994 relative à l'emploi de la langue française), destinadas a garantizar la primacía del uso de los términos tradicionales franceses sobre los anglicismos. En la misma línea, la Académie française participa, junto con las comisiones especializadas, en trabajos que proponen términos franceses en diversos campos para designar nuevos conceptos y realidades, actuando como miembro de oficio de la Comisión General que se encarga de examinar las propuestas de dichas comisiones y da su aprobación para la publicación en el Diario Oficial de los términos, acompañados de sus definiciones. El uso de equivalentes franceses se convierte entonces en obligatorio dentro de las administraciones y servicios públicos, sustituyendo a los términos extranjeros.

Pese a ello, la evolución natural del lenguaje, los efectos de la globalización y el protagonismo adquirido por los mass media en la sociedad, han promovido la difusión de los términos ingleses también en la lengua francesa. Además, los periódicos adoptan cada vez más elementos del lenguaje hablado produciéndose una oralización de la palabra escrita para crear una ilusión de familiaridad con los lectores (Tuomarla,1999) y viceversa, el lenguaje periodístico tiene una gran influencia en la sociedad.

En suma, como recoge Le Parisien (2016) en referencia a los anglicismos: «vous pouvez les aimer ou ne pas les aimer, mais vous ne pouvez pas vous en débarrasser»». 
En lo que respecta al trabajo de investigación desarrollado en torno a la prensa francesa, se han identificado los siguientes anglicismos:

\begin{tabular}{|c|c|c|c|}
\hline Le Parisien & Le Monde & L'Échos & L'Équipe \\
\hline booster & aware & big data & champions \\
building & backoffice & business & club \\
gallery & best-seller & bonus & coach \\
interview & best-of & break even & drive \\
lobby & brand & burnout & game \\
low-cost & backstage & cash & handball \\
meeting & freelance & feedback & hooligan \\
musthave & liker & leader & green \\
network & location & leasing & kick \\
staff & open space & meeting & kit \\
sponsor & peep-show & network & last minute \\
story-board & talk-show & new economy & putt \\
zoom & workflow & sitcom & sponsoring \\
& & summit & star \\
& & timing & strike \\
& & roadmap & team \\
& & venture & trial \\
& & workflow & \\
& & welfare & zoom \\
& & & \\
& & &
\end{tabular}

Tabla 3. Anglicismos presentes en las noticias más relevantes de Le Parisien, Le Monde, L'Échos y L'Equipe durante el año 2018 (Elaboración propia).

\subsection{España}

De acuerdo con Rodríguez González (1999), se pueden distinguir en España tres fases en la inclusión del léxico inglés en el lenguaje español. La primera fase se inicia durante el siglo XVIII hasta la primera mitad del siglo XIX, un periodo en el que se intensifican las relaciones entre España e Inglaterra debido a conflictos bélicos, existiendo por tanto un contacto entre ambas lenguas. La segunda fase corresponde al periodo de la Revolución Industrial, en el que al igual que ocurrió en Francia e Italia, se hace patente la hegemonía del inglés en España, tanto en la lengua como en la cultura, la economía, la ciencia y el comercio. La tercera fase al igual que los anteriores países analizados, se establece a partir de la Segunda Guerra Mundial. Aunque a diferencia de Italia y Francia, nuestro país no formó parte de dicho conflicto bélico internacional, sufrió las consecuencias asociadas al mismo, entre ellas, el impacto de la cultura estadounidense en la sociedad española y a nivel mundial, y la consiguiente incorporación de anglicismos a nuestra lengua. 
«Todos los medios de comunicación son una reconstrucción, un modelo de alguna capacidad biológica acelerada más allá de la capacidad humana de llevarla a cabo (...)» (McLuhan, 1978: 94) ${ }^{3}$ y, dicha capacidad es la responsable de la rápida extensión de la información y el incremento acelerado de la penetración de términos ingleses en nuestra realidad lingüística. Si bien la introducción de préstamos ingleses en la lengua española se ha desarrollado de forma más tardía, queda patente en la prensa y en los medios de comunicación españoles, se ha identificado una curva ascendente desde finales del siglo $X X$ hasta la actualidad, llegando incluso a incorporarse poco a poco en los diccionarios académicos, «muestra de que estos vocablos han sido normalizados en la lengua receptora» (Vázquez-Amador, 2015: 54).

Con base en nuestro trabajo de investigación, la prensa española ha dado como resultado la recopilación de los anglicismos que se enumeran a continuación:

\begin{tabular}{|c|c|c|c|}
\hline EI Mundo & El País & El Economista & Marca \\
\hline best seller & briefing & best-seller & jogging \\
blockbuster & computing & broker & leader \\
call center & coworker & break & mister \\
cloud computing & crack & display & rally \\
crowdfunding & fan & ranking \\
food truck & feedback & light & round \\
glamour & handicap & lobby & play-off \\
hashtag & hardware & light & set \\
lobby & hacker & ranking & shock \\
malware & hit & rating & slogan \\
remake & hobby & & sport \\
roaming & look & & \\
spoiler & malware & & \\
selfie & mindfulness & & \\
shock & naming & & \\
showroom & pub & & \\
smartphone & ranking & & \\
start-up & set & \\
sandwich & & & \\
\hline
\end{tabular}

Tabla 4. Anglicismos presentes en las noticias más relevantes de El Mundo, El Pais, El Economista y Marca, durante el año 2018 (Elaboración propia).

${ }^{3}$ En Marín, D. F. A., y Pérez, F. J. S. (2003). Tecnologías de ayuda en personas con trastornos de comunicación. Nau Llibres. 


\subsection{Análisis contrastivo}

De estos datos anteriores, complementados por el proceso documental llevado a cabo, se derivan las siguientes conclusiones:

- En lo que respecta a la frecuencia de uso, hemos detectado que la lengua italiana es la que mayor número de anglicismos ha presentado en todos los diferentes periódicos que conforman la muestra, identificándose una clara paridad en los resultados. A pesar de contar con equivalentes en la lengua materna, prima el uso de anglicismos arraigados en el léxico italiano (p. ej.: «manager», cuya traducción al italiano sería uomo d'affari, capo o gestore, se utiliza con mayor preferencia el término en inglés, en muchos casos como préstamo integrado, tanto en noticias de tipo general (privacy, premier, leader, break, report, etc.), como en prensa más especializada (format, import, size, surplus, mister, coach, competitor, etc.). Ello pone de manifiesto el uso de anglicismos por esnobismo (Marazzini y Petralli, 2015). Asimismo, existe cierta paridad en el número de resultados obtenidos en cada periódico analizado y se observó cierta repetición de los términos en diferentes noticias.

- La presencia de voces inglesas en el caso de la prensa francesa es mayor de la esperada, e igualmente se suelen utilizar para denominar a conceptos que existen en la lengua francesa (p. ej.: meeting: réunion; musthave: essentielle, network: réseau). En la prensa española, la presencia de términos ingleses parece haberse visto incrementada en los últimos años, observándose en los resultados de la investigación un gran número de voces inglesas. Al igual que en italiano y francés, los anglicismos describen realidades que ya cuentan con equivalente en español, pero también destaca, principalmente en la prensa española, la presencia de neologismos que solo tienen referente léxico en inglés (p. ej: spoiler o hashtag).

- Con respecto a la temática, se observa en italiano y francés, una mayor presencia de préstamos ingleses en la prensa económica y deportiva, con repetición de los términos en diferentes noticias, aunque la presencia en la prensa general es mayor de la esperada. En la prensa española, destaca la presencia de términos ingleses en noticias de carácter general, siendo superior a los resultados obtenidos en El Economista o Marca. No es casual la intensificación en el uso de voces inglesas en áreas semánticas en las que los países anglo-hispanos son más poderosos. Una realidad que queda comprobada mediante el análisis de los resultados anteriores, donde podemos identificar una mayor carga terminológica en dichos diarios en comparación con los de temática general, así como un incremento de neologismos. Sin embargo, reiteramos que, según los resultados de los 
periódicos consultados en 2018 de la prensa española, parece haberse desarrollado más rápidamente el uso de términos ingleses en la vida cotidiana en comparación con los anglicismos encontrados en periódicos especializados.

Durante el proceso documental, se observó además que las noticias de las secciones del periódico tales como sucesos, local o de sociedad presentaban un número de anglicismos bastante inferior o en ocasiones incluso nulo, en los tres idiomas de trabajo; mientras que noticias relacionadas con el cine, la música, la moda o las redes sociales, mostraban una mayor presencia de anglicismos como evidencia de la transferencia cultural anglosajona.

\section{Conclusión}

«The new electronic interdependence recreates the world in the image of a global village» (McLuhan, 1962).

El trabajo de investigación reveló que el inglés ha entrado a formar parte indiscutible de las lenguas romances objeto de estudio (italiano, francés y español), aunque existe una diferencia ente ellas en el grado de intensidad e incursión, considerándose el italiano la lengua que mayor presencia de anglicismos presenta, seguida del francés y después del español. El incremento en la frecuencia de uso de los anglicismos parece estar asociado a la condición de cierto prestigio que parece otorgar la recurrencia a términos ingleses en el lenguaje general y especializado. Además de este particular estatus asociado a los anglicismos, se comprueba también otra de las premisas de nuestro estudio: la existencia de una relación directamente proporcional con el número de voces en inglés en un área de conocimiento determinada y el grado de dominio de dicha rama de especialidad por parte de un país anglosajón.

\section{Referencias bibliográficas}

Cartago, G. (1994). L'apporto inglese. Storia della lingua italiana, (3, 72I-750.

Coco A. (2003). Briciole di linguistica. En Giovanardi Claudio, Gualdo Riccardo, Inglese - italiano I a I Tradurre o non tradurre le parole inglesi?, Manni: Lecce, 28-52;

Crystal, D. (1997). English as a global language. Cambridge.

Italiano, G. (1999). Parole a buon rendere ovvero l'invasione dei termini angloitaliani , Cadmo: Firenze.

Marazzini, C., y Petralli, A. (Eds.). (20I5). La lingua italiana e le lingue romanze di fronte agli anglicismi. goWare. 
Matras, Y. (20|3). Languages in contact in a world marked by change and mobility. Revue française de linguistique appliquée, I8(2), 7-I3.

McLuhan, M. (1962). The Making of Typographic Man. Toronto: University of Toronto Press, (18).

Rodríguez González, F. (1999). Anglicisms in Contemporary Spanish. An Overview. Atlantis, (2I), I03-I39.

Saugera, V. (2017). La fabrique des anglicismes. Travaux de linguistique, (2), 59-79.

Snell-Hornby, M. (2000). Communicating in the Global Village: On Language, Translation and Cultural Identity. En Translating in the Global Village. Ed. Christina Schäeffner. Clevedon, Buffalo, Toronto, Sydney: Multilingual Matters. II-28.

Vázquez Amador, M. (20I5). Estudio descriptivo de la influencia del inglés en la prensa mexicana del siglo XIX. Tonos digital, (29) 3I-57.

Zethsen, K. (2010). Has Globalisation Unburdened the Translator? Meta: journal des traducteurs/Meta: Translators' Journal, 55(3), 545-557. 